\title{
Photospheric properties and fundamental parameters of $M$ dwarfs
}

\author{
A. S. Rajpurohit ${ }^{1}$, F. Allard ${ }^{2}$, G. D. C. Teixeira ${ }^{3,4}$, D. Homeier ${ }^{5}$, S. Rajpurohit ${ }^{6}$, and O. Mousis ${ }^{7}$ \\ 1 Astronomy \& Astrophysics Division, Physical Research Laboratory, Ahmedabad 380009, India \\ e-mail: arvindr@prl.res.in \\ 2 Univ. Lyon, ENS de Lyon, Univ. Lyon1, CNRS, Centre de Recherche Astrophysique de Lyon, UMR 5574, 69007 Lyon, France \\ 3 Instituto de Astrofísica e Ciências do Espaço, Universidade do Porto, CAUP, Rua das Estrelas, 4150-762 Porto, Portugal \\ ${ }^{4}$ Departamento de Física e Astronomia, Faculdade de Ciências, Universidade do Porto, Rua Campo Alegre, 4169-007 Porto, Portugal \\ 5 Zentrum für Astronomie der Universität Heidelberg, Landessternwarte, Königstuhl 12, 69117 Heidelberg, Germany \\ 6 Clausthal University of Technology, Institute for Theoretical Physics, Leibnizstr. 10, 38678 Clausthal-Zellerfeld, Germany \\ 7 Aix-Marseille Université, CNRS, LAM (Laboratoire d'Astrophysique de Marseille), UMR 7326, 13388 Marseille, France
}

Received 4 July 2017 / Accepted 21 August 2017

\begin{abstract}
Context. M dwarfs are an important source of information when studying and probing the lower end of the Hertzsprung-Russell (HR) diagram, down to the hydrogen-burning limit. Being the most numerous and oldest stars in the galaxy, they carry fundamental information on its chemical history. The presence of molecules in their atmospheres, along with various condensed species, complicates our understanding of their physical properties and thus makes the determination of their fundamental stellar parameters more challenging and difficult.

Aims. The aim of this study is to perform a detailed spectroscopic analysis of the high-resolution $H$-band spectra of $\mathrm{M}$ dwarfs in order to determine their fundamental stellar parameters and to validate atmospheric models. The present study will also help us to understand various processes, including dust formation and depletion of metals onto dust grains in $\mathrm{M}$ dwarf atmospheres. The high spectral resolution also provides a unique opportunity to constrain other chemical and physical processes that occur in a cool atmosphere.

Methods. The high-resolution APOGEE spectra of M dwarfs, covering the entire $H$-band, provide a unique opportunity to measure their fundamental parameters. We have performed a detailed spectral synthesis by comparing these high-resolution $H$-band spectra to that of the most recent BT-Settl model and have obtained fundamental parameters such as effective temperature, surface gravity, and metallicity $\left(T_{\text {eff }}, \log g\right.$, and $\left.[\mathrm{Fe} / \mathrm{H}]\right)$, respectively.

Results. We have determined $T_{\text {eff }}, \log g$, and $[\mathrm{Fe} / \mathrm{H}]$ for $45 \mathrm{M}$ dwarfs using high-resolution $H$-band spectra. The derived $T_{\text {eff }}$ for the sample ranges from 3100 to $3900 \mathrm{~K}$, values of $\log g$ lie in the range $4.5 \leq \log g \leq 5.5$, and the resulting metallicities lie in the range $-0.5 \leq[\mathrm{Fe} / \mathrm{H}] \leq+0.5$. We have explored systematic differences between effective temperature and metallicity calibrations with other studies using the same sample of M dwarfs. We have also shown that the stellar parameters determined using the BT-Settl model are more accurate and reliable compared to other comparative studies using alternative models.
\end{abstract}

Key words. stars: atmospheres - stars: fundamental parameters - stars: low-mass - stars: late-type

\section{Introduction}

The lower end of the Hertzsprung-Russell (HR) diagram has proven extremely useful in the last few decades as most of the very-low-mass stars (VLM) in the Galaxy are located in that region. Seventy percent of the Galactic stellar population (Bochanski et al. 2010) consists of these VLMs, in particular M dwarfs, and they contribute approximately $40 \%$ of the total stellar mass budget of the Galaxy (Gould et al. 1996; Mera et al. 1996; Henry 1998). Depending on its metallicity, the mass of any particular $\mathrm{M}$ dwarf ranges from $0.6 M_{\odot}$ to the hydrogenburning limit of about $0.075 M_{\odot}$ (Chabrier et al. 2000). M dwarf populations show great diversity, in that one can find young metal-rich M dwarfs in open clusters, whereas galactic halos (Green \& Margon 1994) and the globular clusters (Cool et al. 1996; Renzini et al. 1996) are known to host metal-poor M dwarfs that are billions of years old. Therefore, $M$ dwarfs are one of the most important stellar components of the Galaxy, carrying fundamental information on the Galaxy's structure, formation, and chemical history. Recently, brown dwarfs and super earths were found around M dwarfs (Bonfils et al. 2012;
Anglada-Escudé et al. 2016; Gillon et al. 2017) which makes them an important laboratory to study and understand their formation.

Despite the large number of $\mathrm{M}$ dwarfs in the Galaxy, a homogenous sample, in terms of age and metallicity, is very difficult to obtain, as high-resolution images and good signal-tonoise ratio spectra are rare because of their intrinsics faintness. Moreover, the non-existence of true continuum makes it difficult or impossible to isolate different spectral diagnostics and to disentangle the effective temperature, surface gravity, and metallicity $\left(T_{\text {eff }}, \log g\right.$, and $\left.[\mathrm{Fe} / \mathrm{H}]\right)$. The presence of diatomic and triatomic molecules along with dust in $\mathrm{M}$ dwarf atmospheres, as we go from early to late $\mathrm{M}$ dwarfs, makes access to the spectral continuum nearly impossible both in the optical and in the nearinfrared (NIR). Nevertheless, because of their cool temperature and low metal content, $\mathrm{M}$ dwarfs provide the best laboratory to study the dust and cloud formation as well as radiative transfer in their atmosphere.

As the $T_{\text {eff }}$ of $\mathrm{M}$ dwarfs decreases from early to late M dwarfs, the optical and NIR spectra of M dwarfs indicate large numbers of diatomic $(\mathrm{SiH}, \mathrm{CaH}, \mathrm{TiO}, \mathrm{VO}, \mathrm{CrH}, \mathrm{FeH}, \mathrm{OH}$, 
$\mathrm{CO})$ and triatomic $\left(\mathrm{CaOH}, \mathrm{H}_{2} \mathrm{O}\right)$ molecules. The Rayleigh-Jeans branch of $\mathrm{M}$ dwarfs spectral energy distribution (SED) in IR $(<1.3 \mu \mathrm{m})$ is dominated by the $\mathrm{H}_{2} \mathrm{O}$ and $\mathrm{CO}$ molecular absorption bands, whereas in the corresponding optical part $(>0.4 \mu \mathrm{m})$ to NIR $(<1.3 \mu \mathrm{m})$ their SED is governed by TiO, VO and metal hydrides. Due to the presence of these complex and crowded band structures, access to true continuum is not possible, and thus a pseudo-continuum is created, which usually shows the strongest and often resonant atomic lines at lower resolution (Allard 1990; Allard \& Hauschildt 1995). In cooler M dwarfs with spectral type M6 or later, the outermost temperatures of their atmospheres are cool enough to form dust and clouds (see e.g., Tsuji et al. 1996b,a; Allard et al. 1997, 1998; Ruiz 1997). These various physical and chemical processes complicate the understanding of their cool atmospheres, thus making determination of their stellar properties even more difficult.

The proper classification of $\mathrm{M}$ dwarf spectra requires the comparison of a grid of synthetic spectra with observations. These comparisons can thus be used to derive M dwarfs' fundamental parameters. Such comparisons also help to disentangle and quantify basic physical properties and fundamental parameters such as $T_{\text {eff }}, \log g$, and $[\mathrm{Fe} / \mathrm{H}]$. Thus far, $T_{\text {eff }}, \log g$, and $[\mathrm{Fe} / \mathrm{H}]$ ) have not been determined for $\mathrm{M}$ dwarfs with great accuracy. Different groups have used various traditional techniques to estimate the $T_{\text {eff }}$ of $\mathrm{M}$ dwarfs based on broadband photometry and black-body approximations. These relatively old, traditional techniques are not as reliable because the true continua of cool $\mathrm{M}$ dwarfs is embedded in complex and broad molecular absorptions. Furthermore, in $\mathrm{M}$ dwarf atmospheres, the complexity increases significantly as dust and cloud formation occurs with decreasing $T_{\text {eff }}$. In the optical part of their SEDs, this can be seen as the weakening of TiO-, VO-, $\mathrm{CaH}-$, and $\mathrm{CaOH}-$ band opacities by dust Rayleigh scattering, whereas as in the infrared (IR) region, the weakening of water bands occurs due to the greenhouse effect (Allard et al. 2001) or dust back-warming.

Models of the atmospheres of cool, low-mass stars and substellar objects have been the subject of tremendous development in recent decades (Brott \& Hauschildt 2005; Helling et al. 2008; Allard et al. 2012, 2013). Because of this advancement, a number of studies is being carried out to derive the accurate stellar parameters of VLM stars and brown dwarfs using both optical and NIR observations (Burgasser \& Kirkpatrick 2006; Bayo et al. 2014, 2017; Rajpurohit et al. 2012, 2013, 2014, 2016). Bayo et al. $(2017,2014)$ show the importance of consistent fundamental parameters by estimating their atmospheric parameters from optical and in the NIR with lowresolution spectra and photometry of $\mathrm{M}$ dwarfs, simultaneously. Through revised solar abundances by Asplund et al. (2009) and Caffau et al. (2011), and by incorporating updated atomic and molecular line opacities which govern the SED of M dwarfs, atmospheric models such as the BT-Settl (Allard et al. 2013) have seen major improvements in modeling various complex molecular absorption bands. These updated models now also include dust and cloud formation (Allard et al. 2013; Baraffe et al. 2015), which is important for cool $\mathrm{M}$ dwarfs and metal-poor M subdwarfs (sdM), and thus yield promising results which explain the stellar-to-substellar transition and confirm the work of Rajpurohit et al. (2012).

In comparison to our Sun, the determination of atmospheric parameters for $\mathrm{M}$ dwarfs is very different and challenging. Stellar parameters, such $T_{\text {eff }}$, of $\mathrm{M}$ dwarfs remain model-dependent to some extent. There have been many attempts to derive the $T_{\text {eff }}$ scale of $\mathrm{M}$ dwarfs with respect to constant age and metallicity. Due to the lack of very reliable model atmospheres in the past, Bessell (1991) used indirect methods to derive the $T_{\text {eff }}$ scale of M dwarfs based on black-body fitting to the NIR bands, whereas Wing \& Rinsland (1979) and Veeder (1974) fitted much cooler black body to the optical. Tsuji et al. (1996b) and Casagrande et al. (2008) provided a good $T_{\text {eff }}$ determination using an infrared flux method (IRFM) for dwarfs including $\mathrm{M}$ dwarfs. The $\mathrm{M}$ dwarfs in the Rayleigh Jeans tail (mostly red-wards of $2.5 \mu \mathrm{m}$ ) carry little flux compared to black body, thus the IRFM method tends to underestimate $T_{\text {eff }}$ for $M$ dwarfs. Boyajian et al. (2012) used another approach which is based on interferometrically determined radii and bolometric fluxes from photometry to calculate the $T_{\text {eff }}$ for nearby $\mathrm{K}$ and $\mathrm{M}$ dwarfs, whereas Mann et al. (2015) determined the radius and mass by combining the empirical mass-luminosity relationships with evolutionary models, which in turn depend on the $T_{\text {eff }}$ and metallicity.

Recently, Rajpurohit et al. (2013) determined the $T_{\text {eff }}$ of nearby bright $\mathrm{M}$ dwarfs from the low-resolution spectra observed in the visible wavelength using the updated BT-Settl model atmosphere. Their study shows that these updated models can now reproduce the slope of their SED very well, unlike previous studies by Leggett et al. (1996, 1998, 2000, 2001) using previous versions of these models, which were using incomplete opacities and other inaccuracies. The $\log g$ of $\mathbf{M}$ dwarfs can be determined with the help of high-resolution spectra (Passegger et al. 2016; Rajpurohit et al. 2016). Passegger et al. (2016), Rajpurohit et al. (2016) used gravity-sensitive features such as $\mathrm{Na} \mathrm{I}, \mathrm{K} \mathrm{I}$, and $\mathrm{Ca}$ I lines to determine the surface gravity in the optical. Other authors used interferometry to determine the angular diameter of the $\mathrm{M}$ dwarfs, together with mass-luminosity relations to derive the mass and $\log g$ (e.g. Ségransan et al. 2003).

A proper metallicity calibration for $M$ dwarfs is essential to determine the planet star metallicity relation, which for FGK stars tends towards the super solar metallicities. The metallicity determination of $\mathbf{M}$ dwarfs can be done in two ways: photometric- and spectroscopic-based methods which are limited to the moderate-resolution spectra in the visible (Woolf \& Wallerstein 2006; Woolf et al. 2009), and in the infrared (Mann et al. 2013a, 2014; Terrien et al. 2012; Rojas-Ayala et al. 2010; Newton et al. 2014). The former techniques use $\mathrm{M}$ dwarf photometry in the visible and infrared bands to create $[\mathrm{Fe} / \mathrm{H}]$ calibrations (Bonfils et al. 2005; Johnson \& Apps 2009; Schlaufman \& Laughlin 2010), while the latter ones rely on low- to high-resolution spectra to measure indices and lines in order to establish spectroscopic calibrations or compare them to synthetic spectra, made from M dwarf atmospheric models (Valenti et al. 1998; Bean et al. 2006a,b; Lindgren \& Heiter 2017). Recently Souto et al. (2017) presented the first detailed NIR chemical abundance analysis observed by SDSS-IV-Apache Point Observatory Galactic Evolution Experiment (APOGEE; Majewski et al. 2017). The $T_{\text {eff }}$ values adopted in this study were derived from the photometric calibrations for M dwarfs by Mann et al. (2015) for the $V-J$ and $R-J$ colors.

In this paper, we take advantage of the updated BT-Settl model grid and high-resolution $H$-band spectra to determine the atmospheric parameters $\left(T_{\text {eff }}, \log g\right.$, and $\left.[\mathrm{Fe} / \mathrm{H}]\right)$ of $45 \mathrm{M}$ dwarfs. In Sect. 2, we briefly describe the observations and some aspects of data reduction. In Sect. 3, we describe the BT-Settl model atmosphere used in this study. Section 4 presents the results and describes the comparison with models and determination of stellar parameters. A Summary and Discussion are presented in Sect. 5 . 
A. S. Rajpurohit et al.: $H$-band spectroscopy of M dwarfs

Table 1. NIR photometry for our sample is taken from 2MASS along with their coordinates and spectral types.

\begin{tabular}{|c|c|c|c|c|c|c|}
\hline $\begin{array}{l}\text { 2MASS ID } \\
\text { 2MXXXXX }\end{array}$ & $J$ & $H$ & $K_{\mathrm{s}}$ & $\alpha$ & $\delta$ & SpT \\
\hline $00131578+6919372$ & & $07.98 \pm 0.02$ & $07.74 \pm 0.02$ & 03.315773 & 69.327003 & \\
\hline & & & & & & \\
\hline $00350487+5953079$ & & $.40 \pm 0.02$ & $.16 \pm 0.02$ & & & \\
\hline $01195227+8409327$ & $9.85 \pm 0.026$ & & & & & \\
\hline & & $07.81 \pm 0.01$ & & 2.223315 & & \\
\hline $03152943+5751330$ & & & & & & \\
\hline & & & & & & \\
\hline & & & & & & \\
\hline & & & & & & \\
\hline & & & & & & \\
\hline & & & & & & \\
\hline & & & & & & \\
\hline & & & & & & \\
\hline & & & & & & \\
\hline & & & & & & \\
\hline & & & & & & \\
\hline & & & & & & \\
\hline & & & & & & \\
\hline & & & & & & \\
\hline & & & & & & \\
\hline & & & & & & \\
\hline & & & & & & \\
\hline & & & & & & \\
\hline & & & & & & \\
\hline & & & & & & \\
\hline & & & & & & \\
\hline & & & & & & \\
\hline & & & & & & \\
\hline & & & & & & \\
\hline & & & & & & \\
\hline & & & & & & \\
\hline & & & & & & \\
\hline & & & & & & \\
\hline & & & & & & 15.0 \\
\hline & & & & & & \\
\hline & & & & & & \\
\hline $19071270+4416070$ & & & & & & M4.5 \\
\hline & & & & & & \\
\hline & & & & & & M4.3 \\
\hline & & & & & & \\
\hline & & & & & & \\
\hline $0726+4518089$ & $11.33 \pm 0.023$ & & & 295.7802 & 45.3024 & \\
\hline & & & & & & \\
\hline & & & & & & \\
\hline $21105881+4657325$ & $09.87 \pm 0.022$ & $09.26 \pm 0.01$ & $09.05 \pm 0.01$ & 317.745051 & 46.959034 & \\
\hline
\end{tabular}

\section{Observational data and sample selection}

Regarding the details of the APOGEE survey along with data reduction the reader is referred to (Majewski et al. 2017; Wilson et al. 2010, 2012). The details of APOGEE M dwarfs ancillary project along with target selection and data reduction are described in Deshpande et al. (2013) and Nidever et al. (2015). We obtained spectra of $45 \mathrm{M}$ dwarfs from Deshpande et al. (2013) M dwarfs ancillary project using SDSS-III data release 12 (Alam et al. 2015). The spectral type and photometry are com- piled using Simbad and Vizier catalog access through Centre de Donnees astronomiques de Strasbourg and are given in Table 1.

The presence of broad and complex molecular absorption in $H$-band makes this region one of the most difficult wavelength regimes for identifying various weak atomic absorption features in the spectra of $\mathrm{M}$ dwarfs. The dominant NIR features are due to photospheric absorption by $\mathrm{H}_{2} \mathrm{O}, \mathrm{FeH}, \mathrm{CO}, \mathrm{OH}$, and neutral metals. The absorption lines of neutral metals, as well as the bands of $\mathrm{H}_{2} \mathrm{O}$ and $\mathrm{CO}$, become stronger with decreasing $T_{\text {eff }}$. In the optical region, $\mathrm{M}$ dwarfs show strong features relative to the 

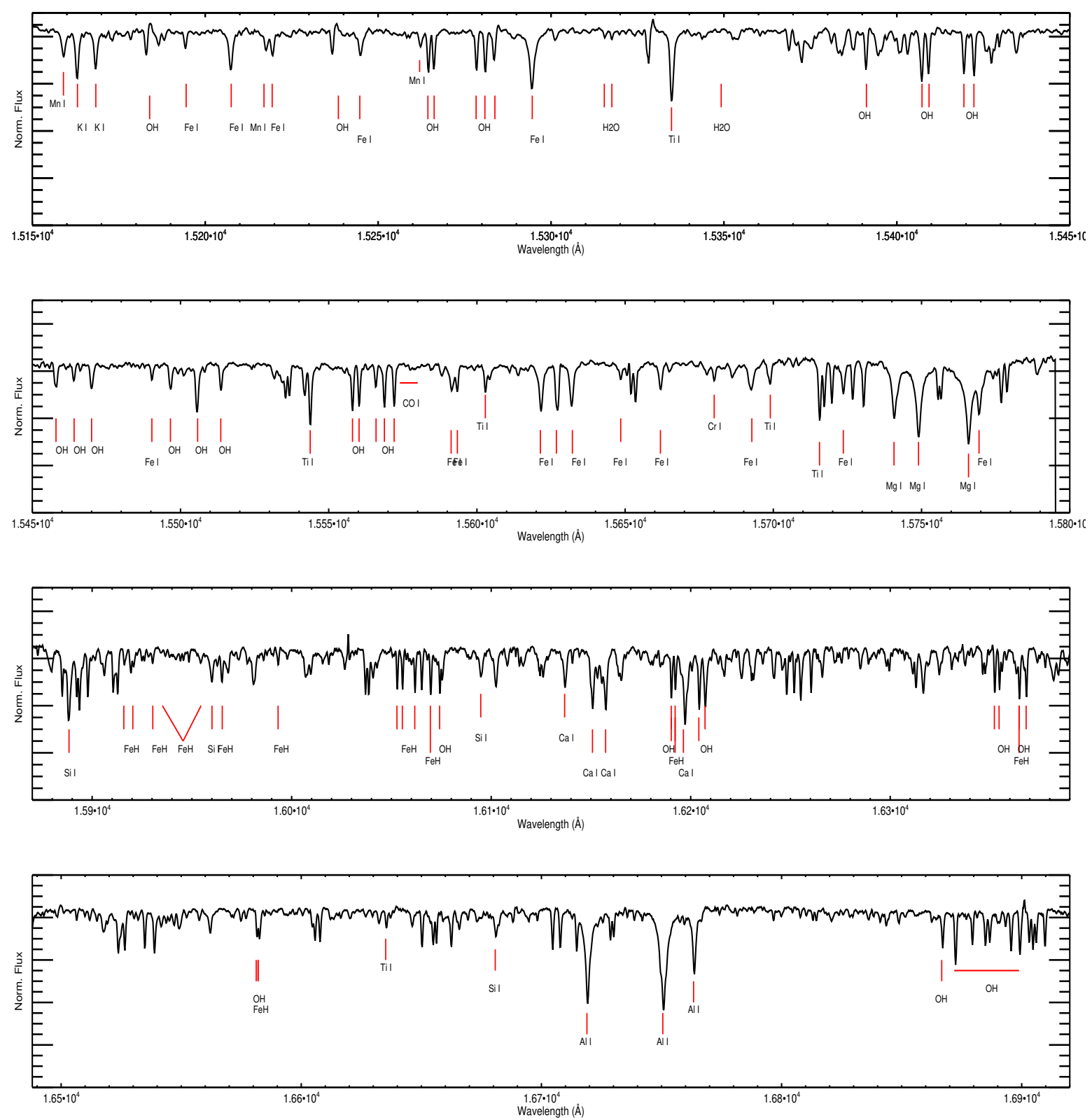

Fig. 1. APOGEE spectra of 2M11091225-0436249 (M0.5). The main spectral features can be seen, including atomic lines such as Fe I, Ca I, Na I, K I, Si I, Mg I, Al II, along with some hydride bands such as those of FeH and OH. We used mainly Souto et al. (2017) for the spectral feature recognition.

strength of the $\mathrm{TiO}$ and $\mathrm{VO}$ molecular bands. However, in the NIR regime, the dominant molecular features are due to $\mathrm{H}_{2} \mathrm{O}$. Also the single metal species such as $\mathrm{FeH}$ will not show the same level of decrease as the double metal TiO. The effect of collisional induced absorption (CIA) by $\mathrm{H}_{2}$ on atomic spectral lines such as those of Fe I, Ca I, Na I, K I, Si I, Mg I, Al I, along with the strengthening of hydride bands such as those on $\mathrm{FeH}$ can be seen in their $H$-band spectra (Fig. 1). As expected from the high $\log g$ of $\mathrm{M}$ dwarfs, the atomic features such as Ca I, $\mathrm{Na}$ I, and $\mathrm{K} \mathrm{I}$ are massively pressure broadened. The $\mathrm{OH}$ and FeH produce more diffuse absorption NIR, unlike TiO and VO which produce distinctive band heads in the optical. The significant opacity source in the $H$-band of $\mathrm{M}$ dwarfs is mainly $\mathrm{FeH}$ but its relative strength decreases and becomes saturated with decreasing temperature. In general one can see various prominent atomic lines such as $\mathrm{Ca}$ I, Na I, K I, Si I, Mg I, and Al I through- out all the observed spectra. However, it is difficult to identify and measure the intensities of these atomic lines in the region where strong molecular absorption features are present.

The Ca I lines at $1.6136 \mu \mathrm{m}, 1.6150 \mu \mathrm{m}$, and $1.6157 \mu \mathrm{m}$, $\mathrm{K}$ I lines at $1.5163 \mu \mathrm{m}$, and $156168 \mu \mathrm{m}, \mathrm{Mg}$ I lines at $1.5740 \mu \mathrm{m}$, $1.5748 \mu \mathrm{m}$, and $1.5765 \mu \mathrm{m}$, and Al I lines at 1.6718, 1.6750, and $1.6763 \mu \mathrm{m}$ can bee seen in all the observed spectra. These atomic lines become broadened as one goes from early to later $\mathrm{M}$ dwarfs. As these atomic lines are so broad, their equivalent widths (EW) are of several angstroms. The strengths of these atomic features depend on various stellar parameters such as $T_{\text {eff }}$, $[\mathrm{Fe} / \mathrm{H}]$, and luminosity. These atomic lines which are relatively free from any blends, and which are not contaminated by telluric lines, are ideal features for studying their sensitivity to various stellar parameters. 

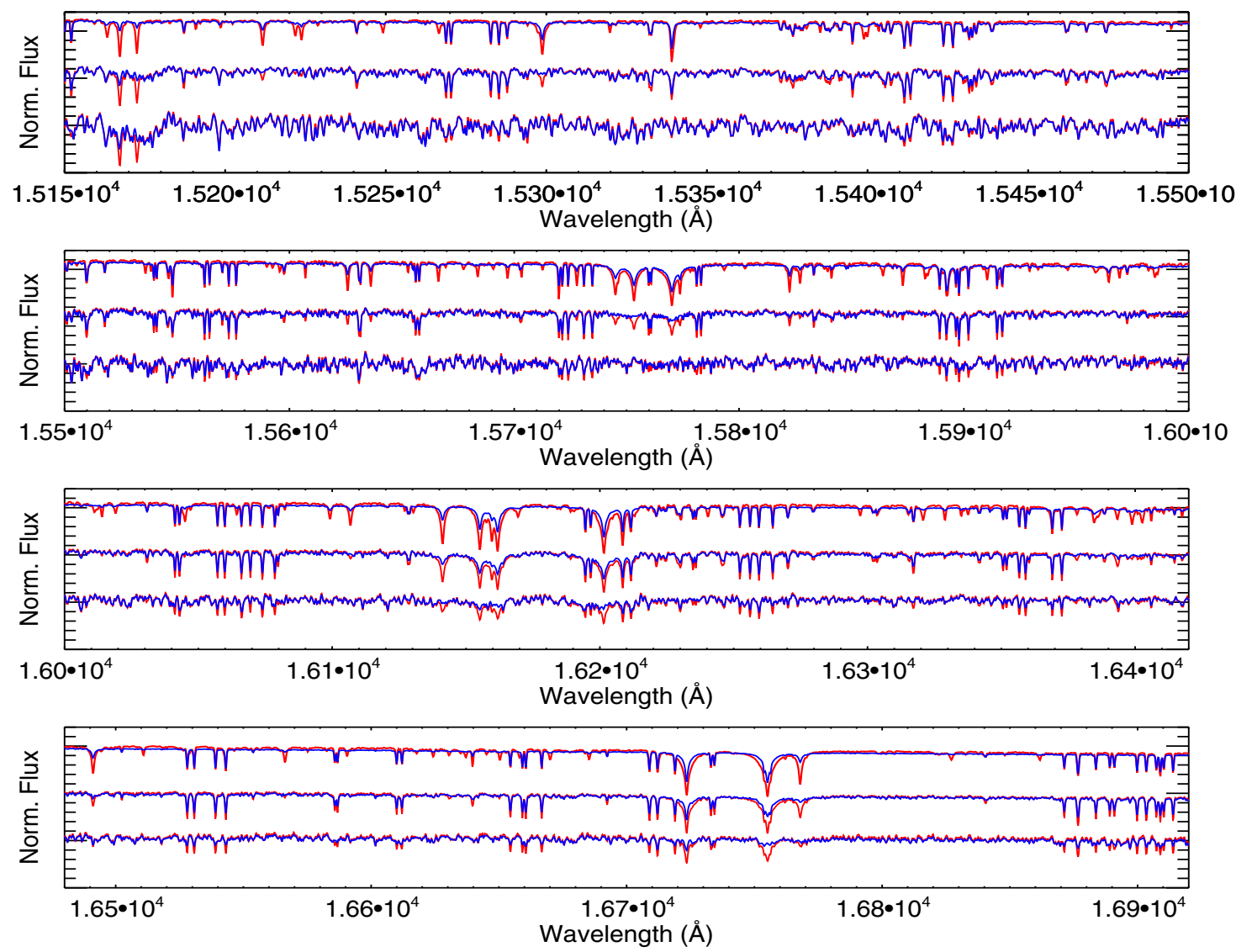

Fig. 2. BT-Settl synthetic spectra from $4000 \mathrm{~K}$ to $3000 \mathrm{~K}$ at a step of $500 \mathrm{~K}$ (top to bottom in each panel) of $H$-band computed with PHOENIX radiative transfer code. The red and blue lines represent the synthetic spectra at $[\mathrm{Fe} / \mathrm{H}]=+0.5$ (red) and -0.5 (blue) for $T_{\text {eff }}=4000 \mathrm{~K}, 3500 \mathrm{~K}$, and $3000 \mathrm{~K}$, at constant $\log g$ of 5.5 .

\section{Models and synthetic spectra}

BT-Settl model atmosphere published by Allard et al. (2012, 2013) is used in this current study. Their computation of these models is performed with the PHOENIX radiative transfer code (Hauschildt et al. 1997; Allard et al. 2001) by assuming the hydrostatic and chemical equilibrium, convection using the mixing-length theory and a sampling treatment of the opacities. The grid of BT-Settl models extends from $T_{\text {eff }} 300$ to $7000 \mathrm{~K}$ in steps of $100 \mathrm{~K}, \log g=2.5$ to 5.5 in steps of 0.5 , and $[\mathrm{M} / \mathrm{H}]=-2.5$ to +0.5 in steps of 0.5 dex, accounting for alphaenhancement and the latest solar abundances by Asplund et al. (2009) and Caffau et al. (2011). The adopted $[\alpha / \mathrm{Fe}]=-0.4 x$ $[\mathrm{Fe} / \mathrm{H}]$ for $-1 \leq[\mathrm{Fe} / \mathrm{H}] \leq 0$ and $[\alpha / \mathrm{Fe}]=+0.4$ for all lower and +0.0 for supersolar metallicities, thus setting the "knee" of the alpha-enrichment relation to an average disk population value. These different $\alpha$ enhancements are mainly for the thin disc and thick disc (Edvardsson et al. 1993; Gratton et al. 1996; Fuhrmann 1998; Adibekyan et al. 2013). At a step of 0.1 dex in $\log g$ and metallicity we have interpolated the grid. The effect of metallicity and $T_{\text {eff }}$ on various atomic and molecular features can bee seen in Fig. 2 with varying $T_{\text {eff }}$ from $4000 \mathrm{~K}$ (top) to $3000 \mathrm{~K}$ (bottom) with a step of $500 \mathrm{~K}$ and $[\mathrm{Fe} / \mathrm{H}]=+0.5$ (red) and -0.5 (blue) at constant $\log g$ of 5.0 in each panel. As found in previous studies (Leggett et al. 1998, 2000), $\log g$ has a relatively small influence on the SED of M dwarfs. But the significant effect of $\log g$ can been seen at high resolution on various atomic line profiles and also on various band systems, whereas metallicity has a large effect on the spectra. We have shown such effects in Fig. 2 where one can see that with decreasing $T_{\text {eff }}$, various atomic features start vanishing and molecular bands begin to dominate; in particular $\mathrm{OH}$ and $\mathrm{FeH}$.

\section{Results}

Comparison with models and determination of stellar parameters. Spectral synthesis using synthetic spectra requires various parameters such as $T_{\text {eff }}, \log g$, and $[\mathrm{Fe} / \mathrm{H}]$ and keeping the Sun as a reference. We followed the same procedure as used in Rajpurohit et al. $(2014,2016)$ to determine $T_{\text {eff }}, \log g$, and $[\mathrm{Fe} / \mathrm{H}]$ using spectroscopic information in $H$-band. The typical $\log g$ of $\mathrm{M}$ dwarfs is approximately $5.0 \pm 0.2$, except for the latest-type M dwarfs (Gizis 1997; Casagrande et al. 2008); we therefore use models with $\log g=4.5-5.5$ for our analysis. To determine the stellar parameters of $\mathrm{M}$ dwarfs in our sample, we performed a $\chi^{2}$ minimization using spectral synthesis employing the new BT-Settl model atmospheres across the entire wavelength range of the observed spectra. No weights are applied in our calculation for different parameters. The synthetic spectral fitting is performed using the following steps: in the first step the synthetic spectra are convolved with an isotropic Gaussian profile with measured instrumental resolution which is then interpolated at each wavelength point of the observed spectra. In the following step we compared the observed spectra with that of the entire grid of models by taking the difference between the flux values of the observed and synthetic spectra at every wavelength point. Then, the sum of the squares of these differences is obtained for each model in the grid, and the best model for each object is selected. We retain the best-match values of $T_{\text {eff }}, \log g$, 

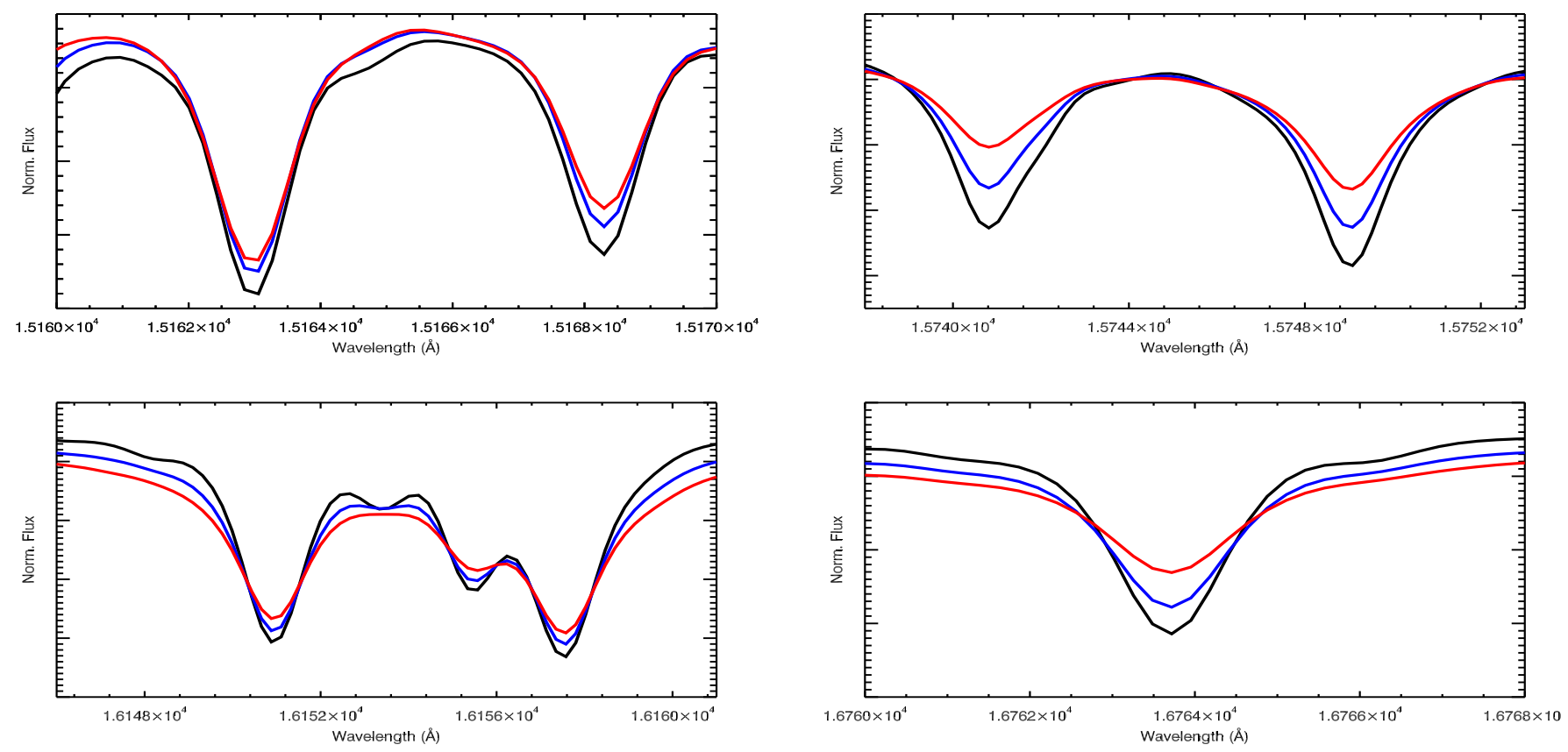

Fig. 3. Effect of $\log g$ on the $\mathrm{K} \mathrm{I}$ (top left), Mg I (top right), Ca I (bottom left), and $\mathrm{Al} \mathrm{I}$ (bottom right) is clearly visible in BT-Settl synthetic spectra at $T_{\text {eff }}=3500 \mathrm{~K}$ and $\log g=4.5$ (black), 5.0 (blue), and 5.5 (red).

and $[\mathrm{Fe} / \mathrm{H}]$ as first guess values on these three parameters. This step of synthetic spectral fitting is performed on the set of models which have not been interpolated to a finer grid in $\log g$, and $[\mathrm{Fe} / \mathrm{H}]$. The comparison is made using a subsample of the model atmosphere grid covering the range of $3000 \mathrm{~K} \leq T_{\text {eff }} \leq 4000 \mathrm{~K}$ in steps of $100 \mathrm{~K},-0.5 \leq[\mathrm{Fe} / \mathrm{H}] \leq 0.5$ in steps of $0.5 \mathrm{dex}$, and $4.0 \leq$ $\log g \leq 5.5$ in steps of 0.5 dex. During this step, we keep all the three parameters $\left(T_{\text {eff }}, \log g\right.$, and $\left.[\mathrm{Fe} / \mathrm{H}]\right)$ free. We excluded the spectral regions from $1.580 \mu \mathrm{m}$ to $1.586 \mu \mathrm{m}$ and from $1.642 \mu \mathrm{m}$ to $1.649 \mu \mathrm{m}$ because of the gap in blue to green and green to red arms of APOGEE.

In the second step, the parameters obtained for each object of our sample from the first step are used as an initial guess value and interpolation is done at a step of $0.1 \mathrm{dex}$ in $\log g$ and $[\mathrm{Fe} / \mathrm{H}]$. Finally, every model of the grid covering the range of $3000 \mathrm{~K} \leq$ $T_{\text {eff }} \leq 4000 \mathrm{~K}$ in steps of $100 \mathrm{~K},-0.5 \leq[\mathrm{Fe} / \mathrm{H}] \leq 0.5$ in steps of $0.1 \mathrm{dex}$, and $4.0 \leq \log g \leq 5.5$ in steps of 0.1 dex are again compared to the observed spectrum at each wavelength point, and the $\chi^{2}$ is calculated to determine the global minima. We retain models that give the lowest $\chi^{2}$ values as the best fit parameters $\left(T_{\text {eff }}, \log g\right.$, and $\left.[\mathrm{Fe} / \mathrm{H}]\right)$ which are shown in Table 2 . In the end, the best models are finally inspected visually by comparing them with the corresponding observed spectra. The uncertainties in Table 2 are based on standard deviation of the derived stellar parameters by accepting $1 \sigma$ variations from the minimum $\chi^{2}$ which in all cases is calculated using constant $\chi^{2}$ boundaries and is based on the $\chi^{2}$ statistic.

We have also checked the behavior of synthetic spectra by visual inspection, looking at the shapes of various atomic species such as Fe I, Ca I, Na I, K I, Si I, Mg I, Al I, and some molecular species such as $\mathrm{OH}, \mathrm{CO}$, and $\mathrm{FeH}$ (for details of the line list, see Souto et al. 2017). The $\mathrm{OH}-$ bands around 1.540 to $1.545 \mu \mathrm{m}$, $1.635 \mu \mathrm{m}$ to $1.636 \mu \mathrm{m}$ and $1.686 \mu \mathrm{m}$ to $1.689 \mu \mathrm{m}$ are insensitive to variations of $0.5 \mathrm{dex}$ in $\log g$ but are rather highly sensitive to $T_{\text {eff }}$. However, at a given $\log g$ they shows huge variation over a change of only $100 \mathrm{~K}$ in $T_{\text {eff }}$. We have conformed our $\log g$ by looking at the width of gravity-sensitive features such as the $\mathrm{K} \mathrm{I}$ $(1.5163 \mu \mathrm{m}$ and $1.5168 \mu \mathrm{m}), \mathrm{Ca} \mathrm{I}(1.6136 \mu \mathrm{m}, 1.6150 \mu \mathrm{m}$, and
$1.6157 \mu \mathrm{m})$, Al I (1.6718 $\mu \mathrm{m}, 1.6750 \mu \mathrm{m}$, and $1.6763 \mu \mathrm{m})$, and $\operatorname{Mg} \mathrm{I}(1.5740 \mu \mathrm{m}, 1.5748 \mu \mathrm{m}$, and $1.5765 \mu \mathrm{m})$ along with the relative strength of metal hydride bands such as FeH. These features are particularly useful gravity discriminants for $\mathbf{M}$ dwarfs and sdM. The overall line strength increases with gravity because of high pressure mainly by $\mathrm{H}_{2}, \mathrm{He}$, and $\mathrm{H} \mathrm{I}$ collisions and due to higher electron pressure on alkali lines (see Fig. 3). The effect of $\log g$ can be seen on width of the damping wings which, in addition, increases (Reiners 2005; Reiners et al. 2016). The effect of metallicity can also be seen on various atomic features where the molecular absorption is less and these atomic features appear clearly. The synthetic spectrum reproduces line profiles of various atomic lines such as Ti, Fe I, Ca I, Mg I, Si I, Mn I, and Al I relatively well. The systematic errors are not eliminated, which arise due to missing or incomplete opacity sources (e.g., FeH-bands and $\mathrm{OH}$ and $\mathrm{CO}$ bands) are not eliminated (Baraffe et al. 2015) and the derived uncertainties are within the derived values of $T_{\mathrm{eff}}, \log g$, and $[\mathrm{Fe} / \mathrm{H}]$.

\section{Summary and discussion}

The high-resolution spectra with good signal-to-noise ratio of $\mathrm{M}$ dwarfs is very important and necessary to determine the $T_{\text {eff }}$, $\log g$, and $[\mathrm{Fe} / \mathrm{H}]$, and also the individual element abundances to a high accuracy. This paper presents the results from the spectral synthesis analysis to determine the fundamental parameters form the high-resolution APOGEE $H$-band spectra for early to mid M dwarfs using the updated BT-Settl model. In the NIR, particularly in $H$-band, the BT-Settl model has never been tested before with the high-resolution spectra of M dwarfs. Therefore, our present study constitutes a benchmark for model atmospheres of low-mass stars in NIR. The physical parameters $T_{\text {eff }}, \log g$, and $[\mathrm{Fe} / \mathrm{H}]$ for stars of our sample is determined by comparing the observed spectra with the synthetic spectra. The main purpose of this paper is to disentangle the parameter space $\left(T_{\mathrm{eff}}, \log g\right.$, and $[\mathrm{Fe} / \mathrm{H}]$ ) with independent information on atmospheric parameters. The comparison of observed spectra with the synthetic spectra is crucial to reveal the inaccuracy or incompleteness of 
Table 2. Stellar parameters of the observed targets determined by minimizing $\chi^{2}$.

\begin{tabular}{|c|c|c|c|}
\hline $\begin{array}{l}\text { 2MASS ID } \\
\text { 2MASS J }\end{array}$ & $\begin{array}{c}\text { This study } \\
T_{\text {eff }} / \log g /[\mathrm{Fe} / \mathrm{H}]\end{array}$ & $\begin{array}{c}\text { Terrien et al. }(2015) \\
T_{\text {eff }},[\mathrm{Fe} / \mathrm{H}] \\
\text { using Mann et al. }(2013 \mathrm{~b}) \\
J, H, \text { and } K \text { calibration }\end{array}$ & $\begin{array}{l}\text { Terrien et al. (2015) } \\
T_{\text {eff }},[\mathrm{Fe} / \mathrm{H}] \\
\text { using Newton et al. (2014) } \\
\text { calibration }\end{array}$ \\
\hline $00131578+6919372$ & $3200 / 5.5 \pm 0.3 /-0.3 \pm 0.04$ & - & - \\
\hline $00321574+5429027$ & $3200 / 5.5 \pm 0.3 /-0.2 \pm 0.04$ & $3366 / 3271 / 3285,-0.03 /-0.08 /-0.05$ & $3206 /+0.00$ \\
\hline $00350487+5953079$ & $3100 / 5.5 \pm 0.3 /-0.0 \pm 0.05$ & - & - \\
\hline $01195227+8409327$ & $3100 / 5.5 \pm 0.3 /-0.3 \pm 0.06$ & - & - \\
\hline $02085359+4926565$ & $3200 / 5.5 \pm 0.3 /-0.1 \pm 0.05$ & $3280 / 3285 / 3330,+0.08 /+0.03 /+0.05$ & $3347 /+0.14$ \\
\hline $03152943+5751330$ & $3200 / 5.5 \pm 0.3 /-0.3 \pm 0.05$ & - & - \\
\hline $3305473+7041145$ & $3200 / 5.5 \pm 0.3 /-0.3 \pm 0.05$ & - & - \\
\hline $03425325+2326495$ & $3200 / 5.5 \pm 0.3 /-0.0 \pm 0.05$ & - & - \\
\hline $4063732+7916012$ & $3100 / 5.5 \pm 0.2 /-0.0 \pm 0.06$ & - & - \\
\hline $04125880+5236421$ & $3100 / 5.5 \pm 0.3 /-0.0 \pm 0.05$ & $3026 / 3304 / 3276,+0.02 /-0.08 /-0.02$ & $-/+0.06$ \\
\hline $05011802+2237015$ & $3200 / 5.5 \pm 0.5 /-0.5 \pm 0.04$ & $-/ 3223 / 3277,+0.21 /+0.03 /+0.12$ & $-/+0.20$ \\
\hline $05030563+2122362$ & $3100 / 5.5 \pm 0.2 /-0.1 \pm 0.07$ & $-/ 3223 / 3277,+0.13 /+0.02 /+0.02$ & $-/+0.05$ \\
\hline $05210188+3425119$ & $3100 / 5.5 \pm 0.3 /-0.5 \pm 0.04$ & - & - \\
\hline $05470907-0512106$ & $3100 / 5.5 \pm 0.3 /-0.3 \pm 0.06$ & - & - \\
\hline $06115599+3325505$ & $3100 / 5.5 \pm 0.2 /-0.1 \pm 0.07$ & $3099 / 3207 / 3276,+0.02 /+0.12 /+0.01$ & $-/+0.13$ \\
\hline $06320207+3431132$ & $3200 / 5.5 \pm 0.3 /-0.4 \pm 0.05$ & $3126 / 3388 / 3313,-0.03 /-0.05 /-0.09$ & $-/+0.03$ \\
\hline $07140394+3702459$ & $3000 / 5.5 \pm 0.2 /-0.5 \pm 0.11$ & - & - \\
\hline $08501918+1056436$ & $3100 / 5.5 \pm 0.2 /-0.0 \pm 0.06$ & - & - \\
\hline $09301445+2630250$ & $3300 / 5.0 \pm 0.5 /-0.3 \pm 0.05$ & $3285 / 3384 / 3359,+0.04 /+0.13 /+0.13$ & $3410 /+0.21$ \\
\hline $10162955+0318375$ & $3200 / 5.5 \pm 0.3 /-0.2 \pm 0.03$ & $3345 / 3399 / 3328,-0.03 /+0.03 /-0.05$ & $3217 /+0.00$ \\
\hline $11005043+1204108$ & $3100 / 5.5 \pm 0.2 /-0.5 \pm 0.11$ & $-/ 3304 / 3276,+0.12 /-0.07 /-0.11$ & $-/+0.05$ \\
\hline $11054316+1014093$ & $3200 / 5.0 \pm 0.5 /-0.0 \pm 0.05$ & $3422 / 3547 / 3418,-0.08 /-0.10 /-0.05$ & $3357 /+0.01$ \\
\hline $11091225-0436249$ & $3900 / 4.5 \pm 0.5 /-0.3 \pm 0.04$ & $3670 / 3786 / 3803,-0.04 /-0.14 /-0.04$ & $3659 /-0.07$ \\
\hline $11474074+0015201$ & $3200 / 5.5 \pm 0.3 /-0.4 \pm 0.04$ & $-/ 3320 / 3300,+0.10 /+0.1 / 5+0.03$ & $-/+0.17$ \\
\hline $12045611+1728119$ & $3200 / 5.5 \pm 0.2 /-0.1 \pm 0.07$ & $3235 / 3318 / 3330,-0.09 /-0.11 /-0.03$ & $3303 /+0.05$ \\
\hline $12232063+2529441$ & $3300 / 5.0 \pm 0.5 /-0.4 \pm 0.04$ & $3267 / 3409 / 3344,-0.05 /-0.01 /+0.05$ & $3303 /+0.05$ \\
\hline $12265737+2700536$ & $3100 / 5.5 \pm 0.3 /-0.0 \pm 0.06$ & $-/ 3304 / 3280,+0.13 /-0.05 /+0.02$ & $-/+0.11$ \\
\hline $13085059+1622039$ & $3200 / 5.5 \pm 0.4 /-0.4 \pm 0.04$ & $3533 / 3545 / 3407,-0.15 /-0.10 /-0.15$ & $-/+0.11$ \\
\hline $13345147+3746195$ & $3200 / 5.5 \pm 0.3 /-0.1 \pm 0.04$ & $3219 / 3348 / 3297,+0.13 /-0.01 /+0.14$ & $-/+0.22$ \\
\hline $13451104+2852012$ & $3200 / 5.0 \pm 0.5 /-0.4 \pm 0.04$ & $3385 / 3441 / 3383,-0.09 /-0.16 /-0.09$ & $3399 /-0.11$ \\
\hline $14592508+3618321$ & $3200 / 5.5 \pm 0.3 /-0.0 \pm 0.04$ & - & - \\
\hline $16370146+3535456$ & $3100 / 5.5 \pm 0.2 /-0.5 \pm 0.04$ & - & - \\
\hline $18451027+0620158$ & $3900 / 4.5 \pm 0.5 /-0.4 \pm 0.04$ & $3707 / 3812 / 3779,+0.03 /+0.07 /-0.03$ & $3664 /-0.05$ \\
\hline $18523373+4538317$ & $3100 / 5.5 \pm 0.2 /-0.0 \pm 0.07$ & $3169 / 3219 / 3285,-0.00 /-0.08 /-0.06$ & $-/-0.03$ \\
\hline $18562628+4622532$ & $3100 / 5.5 \pm 0.3 /-0.0 \pm 0.05$ & $3091 / 3379 / 3307,+0.06 /-0.14 /-0.03$ & $-/+0.05$ \\
\hline $19051739+4507161$ & $3100 / 5.5 \pm 0.3 /-0.2 \pm 0.04$ & $3339 / 3314 / 3313,-0.06 /-0.23 /-0.17$ & $3215 /-0.14$ \\
\hline $19071270+4416070$ & $3100 / 5.5 \pm 0.3 /-0.3 \pm 0.06$ & $3163 / 3269 / 3288,+0.2 / 2-0.0 / 2+0.19$ & $-/+0.25$ \\
\hline $9081576+2635054$ & $3100 / 5.5 \pm 0.3 /-0.4 \pm 0.06$ & $4747 / 3449 / 3280,+0.77 /+0.10 /+0.26$ & $-/+0.29$ \\
\hline $19084251+2733453$ & $3100 / 5.5 \pm 0.3 /-0.2 \pm 0.04$ & $-/ 3368 / 3316,+0.42 /-0.28 /-0.30$ & $3217 /-0.32$ \\
\hline $19321796+4747027$ & $3100 / 5.5 \pm 0.3 /-0.3 \pm 0.05$ & - & \\
\hline $19333940+3931372$ & $3200 / 5.5 \pm 0.3 /-0.1 \pm 0.05$ & - & \\
\hline $19430726+4518089$ & $3100 / 5.5 \pm 0.2 /-0.5 \pm 0.06$ & - & \\
\hline $19443810+4720294$ & $3100 / 5.5 \pm 0.3 /-0.5 \pm 0.04$ & - & \\
\hline $19510930+4628598$ & $3200 / 5.5 \pm 0.3 /-0.0 \pm 0.07$ & $-/ 3279 / 3295,+0.06 /-0.09 /-0.07$ & $-/+0.07$ \\
\hline $21105881+4657325$ & $3300 / 5.0 \pm 0.5 /-0.2 \pm 0.06$ & - & \\
\hline
\end{tabular}

Notes. The uncertainty in $T_{\text {eff }}(\mathrm{K})$ is $\pm 100 \mathrm{~K}$, whereas for $\log g\left(\mathrm{~cm} \mathrm{~s}^{-2}\right)$ and $[\mathrm{Fe} / \mathrm{H}]$ is given below.

the opacities used in the model. The atmospheric parameters derived from the comparison between our sample and the BT-Settl model are summarized in Table 2. For example, Figs. 4 and 5 show the comparison of the best-fit BT-Settl model (red) with the star of spectral type M1.0 and M3.0 (black) in our sample. Their best fit parameters are given in Table 2 . The specific strengths of the $\mathrm{CO}, \mathrm{OH}$, and $\mathrm{FeH}$-band heads are very well reproduced by the synthetic spectra over the entire $\mathrm{M}$ dwarf sequence, showing that the nosy pattern visible at this high spectral resolution is not noise.

The BT-Settl models also predict and reproduce the shape of various atomic lines such as Ca I, Na I, K I, Si I, Mg I, Al I, Ti I rather well and their strengths are well fitted. The observed atomic lines in the spectra are broader and shallower than those predicted by the BT-Settl model in the cooler M dwarfs (spectral type M3 or later). The qualitative behavior of the K I, Al I, Mg I, 

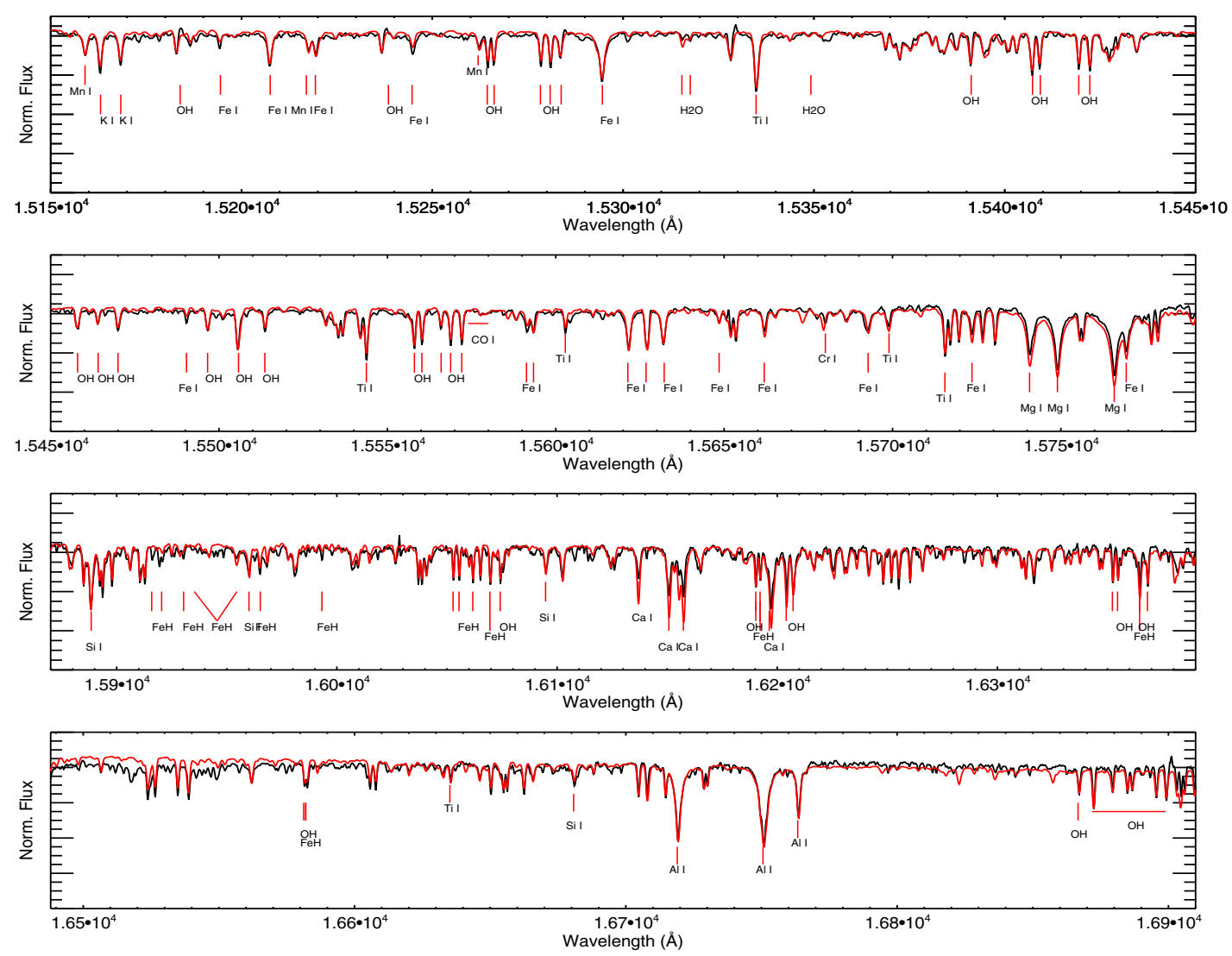

Fig. 4. APOGEE spectra of 2M11091225-0436249 (black) of spectral type M0.5 is compared with the best-fit BT-Settl (red). The best fit value for $T_{\text {eff }}, \log g$, and $[\mathrm{Fe} / \mathrm{H}]$ is $3900 / 4.5 /-0.3$.
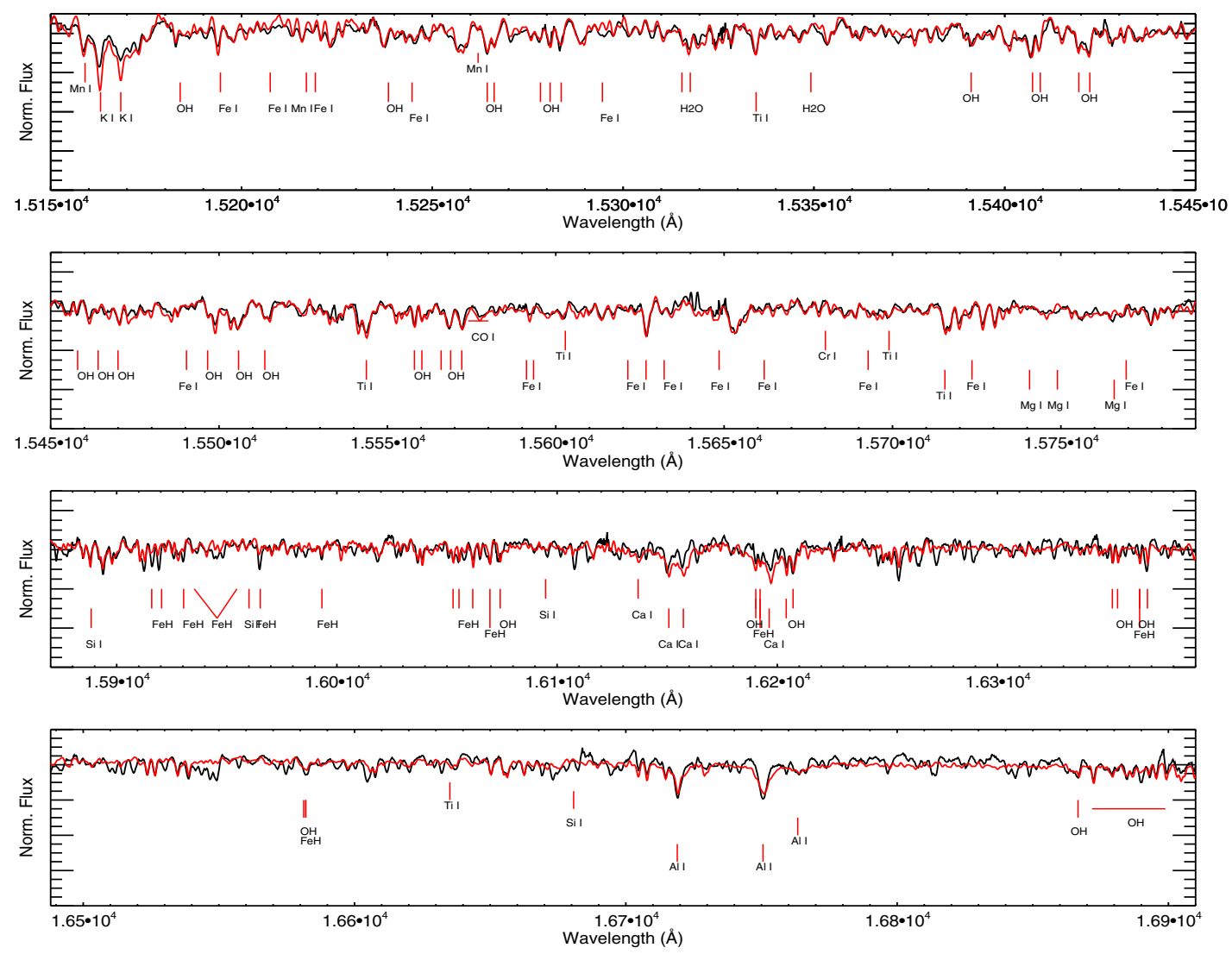

Fig. 5. APOGEE spectra of 2M08501918+1056436 (black) of spectral type M5.0 is compared with the best-fit BT-Settl (red) synthetic spectra. The best fit $T_{\text {eff }}, \log g$, and $[\mathrm{Fe} / \mathrm{H}]$ is $3100 / 5.5 /-0.0$. 

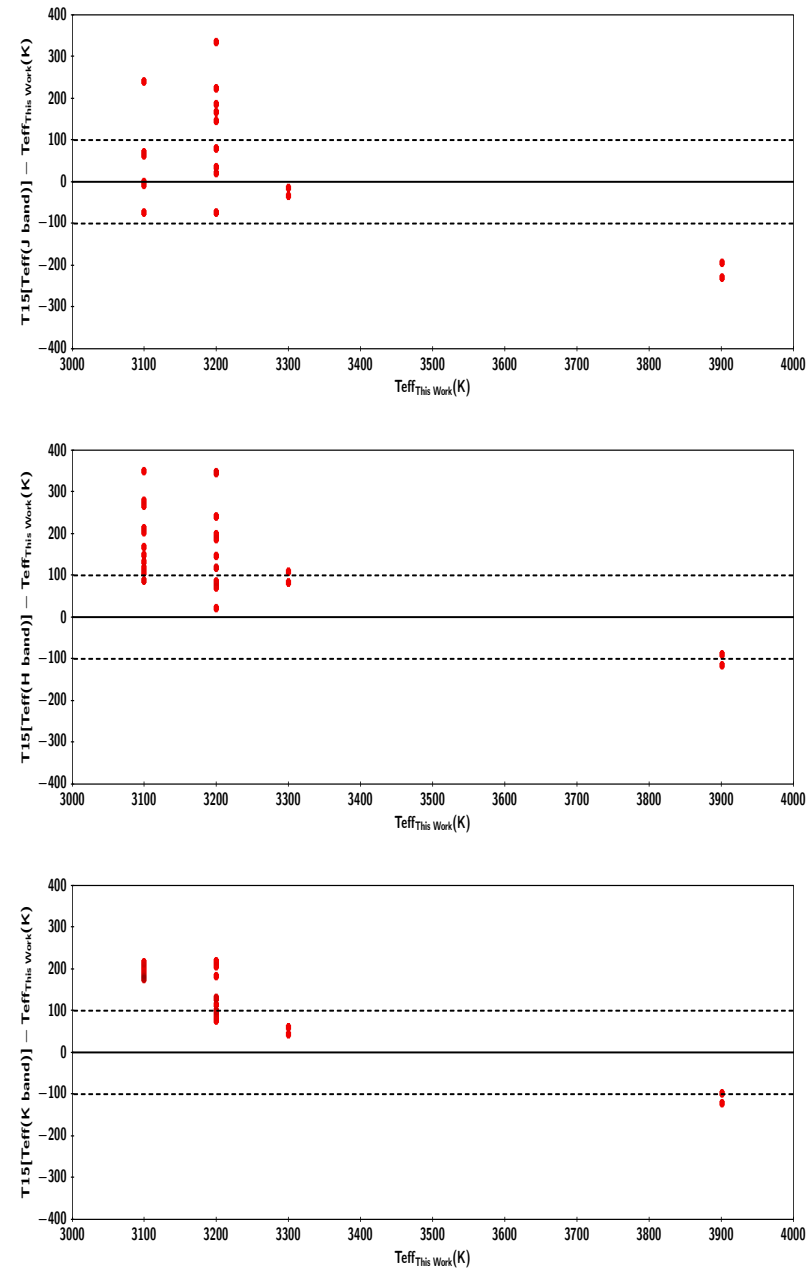

Fig. 6. Difference between the $T_{\text {eff }}$ calibrations from Terrien et al (2015, T15), estimated for the M dwarfs from Mann et al. (2013b) $J$ (top), $H$ (middle), and $K_{\mathrm{s}}$ (bottom) calibrations and $T_{\text {eff }}$ from this work. On the horizontal axis we show the $T_{\text {eff }}$ that we infer from our best fit BT-Settl model used in this work. The black full line represents the origin and the dashed black lines represent the error from the grid size of $100 \mathrm{~K}$.

Ti I, and Ca I lines is well reproduced by the BT Settl model as compared to the strong pressure-broadening wings in the early to mid M dwarfs. In the early M dwarfs, the cores of the observed $\mathrm{K} \mathrm{I}, \mathrm{Al} \mathrm{I}, \mathrm{Mg} \mathrm{I}$, and $\mathrm{Ca} \mathrm{I}$ lines are still visible. The broader absorption component of the atomic lines becomes saturated in $\mathrm{M}$ dwarfs later than M6 which were extending a few tens to one hundred angstroms.

The $T_{\text {eff }}$ is the parameter that causes the largest uncertainty while determining the other stellar parameters of $\mathbf{M}$ dwarfs; their metallicity in particular. Our results for $T_{\text {eff }}$ are in good agreement with the $T_{\text {eff }}$ as a function of spectral type given in Rajpurohit et al. (2013). Now we compare our $T_{\text {eff }}, \log g$, and $[\mathrm{Fe} / \mathrm{H}]$ determination to other works such as Terrien et al. (2015), Schmidt et al. (2016). Terrien et al. (2015) measured the $T_{\text {eff }}$ for the $\mathrm{M}$ dwarfs using color- $T_{\text {eff }}$ relations with the method described by Mann et al. (2013b) along with different temperature indices such as $\mathrm{H}_{2} \mathrm{O}-\mathrm{K} 2$ (Rojas-Ayala et al. 2012), $\mathrm{H}_{2} \mathrm{O}$ $\mathrm{H}$ (Terrien et al. 2012; Mann et al. 2013a). Figure 6 shows the comparison of our measured $T_{\text {eff }}$ with Terrien et al. (2015) which clearly shows that Terrien et al. (2015) overestimates in lower $T_{\text {eff }}$ and underestimates in higher $T_{\text {eff }}$ among the various calibrations using $J, H$, and $K_{\mathrm{s}}$ bands, when compared to our $T_{\text {eff }}$

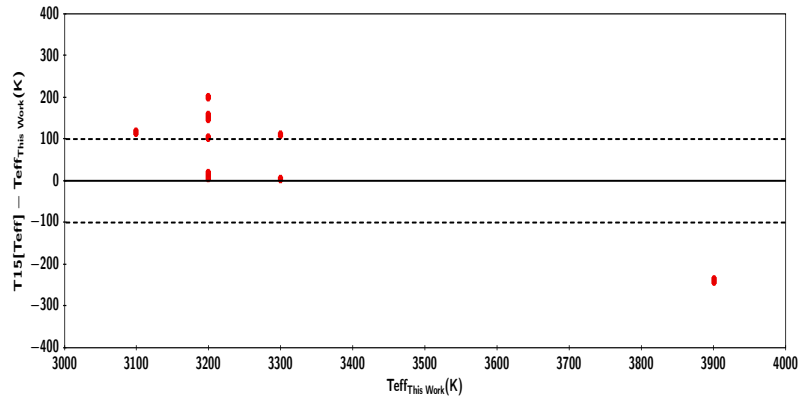

Fig. 7. Difference between the $T_{\text {eff }}$ calibrations from Terrien et al. (2015, T15), estimated for the $\mathrm{M}$ dwarfs from $H$-band relationships given by Newton et al. (2015), calibrations, and $T_{\text {eff }}$ from this work. On the horizontal axis we show the $T_{\text {eff }}$ that we infer from our best fit BT-Settl model used in this work. The black full line represents the origin and the dashed black lines represent the error from the grid size of $100 \mathrm{~K}$.

determinations. This discrepancy could be due to that fact that their determination was based on NIR spectra using the SpeX spectrograph which has significantly lower resolution, and many of their individual determinations were from the $J / H / K$ bands which give relatively inconsistent results. These empirical relations give smaller errors as compared to NIR but they are not as precise as model-fitting techniques. We have also compared the $T_{\text {eff }}$ calculated by Terrien et al. (2015) based on $H$-band atomic feature strengths such as Al I, Mg I, K I, Si I (Fig. 7) using the strength of atomic features studied in Newton et al. (2015). We find an offset of around $200 \mathrm{~K}$ between our $T_{\text {eff }}$ and Terrien et al. (2015) which could be due to the fact that Newton et al. (2015) used a limited number of atomic lines for equivalent width in their analysis where the accurate continuum placement could be the issue.

In four of the stars common eemmen to both ours and the Schmidt et al. (2016) sample, we find that for stars 2MASSJ 11091225-0436249 and 2MASSJ 18451027+0620158, the $T_{\text {eff }}$ by Schmidt et al. (2016) is 200 to $300 \mathrm{~K}$ lower than our measurements, whereas for 2MASSJ $19333940+3931372$ and 2MASSJ $21105881+4657325$ the $T_{\text {eff }}$ by Schmidt et al. (2016) is 200 to $300 \mathrm{~K}$ higher. Schmidt et al. (2016) determine the $T_{\text {eff }}, \log g$, and $[\mathrm{Fe} / \mathrm{H}]$ of late-K and early-M dwarfs selected from the APOGEE spectroscopic survey using APOGEE Stellar Parameters and Chemical Abundances Pipeline (ASPCAP; García Pérez et al. 2016). ASPCAP uses APOGEE ATLAS9 models (Mészáros et al. 2012). For this same set of four targets, we have compared $\log g$ and $[\mathrm{Fe} / \mathrm{H}]$ with $\mathrm{Schmidt}$ et al. (2016) and found a systematic offset of around $0.5 \mathrm{dex}$ to 1.0 dex. We have also compared the best-fit BT-Settl model (red) and MARCS model (blue) with observed spectra of 2M11091225-0436249 and 2M08501918+1056436 (back). We have chosen the identical atmospheric parameters for MARCS model as mentioned in Table 2. We obtain the MARCS (Gustafsson et al. 2008) model which was calculated in 2012 and distributed on the MARCS website ${ }^{1}$. It is clear from Figs. 8 and 9 that in the MARCS model, many $\mathrm{OH}, \mathrm{CO}$, and $\mathrm{FeH}$-bands are missing. Also, the line strength of various atomic species, such as K I, Ti I, Ca I, and Al I, is weaker in the MARCS model than in the BT-Settl model which could be due to low-resolution flux samples as provided on the MARCS website. This discrepancy may also be due to the use of somewhat different assumptions concerning convection, and input data such as contin-

1 http://marcs.astro.uu.se 

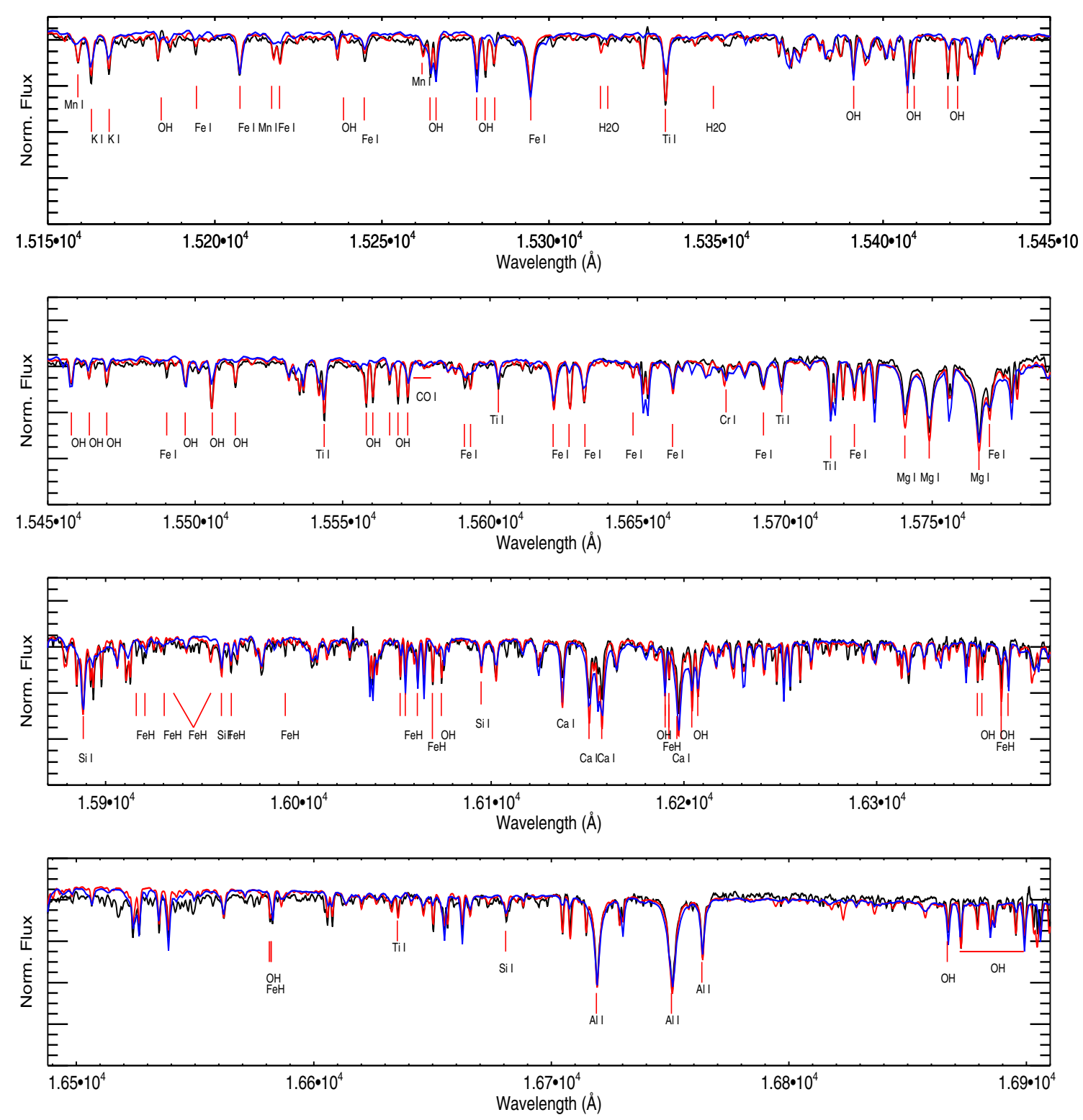

Fig. 8. APOGEE spectra of 2M11091225-0436249 (black) of spectral type M0.5 is compared with the best-fit BT-Settl (red) and MARCS model (blue). The best fit value for $T_{\mathrm{eff}}, \log g$, and $[\mathrm{Fe} / \mathrm{H}]$ is $3900 / 4.5 /-0.3$.

uous opacities in MARCS and ATLAS9 models as compared to the BT-Settl model. Exploring such effects is beyond the scope of this study but a proper way would be to compare the best fit parameters derived using different sets of models, which would provide information on model systematics. For the BTSettl model, a crucial test is to check its consistent accuracy.

Metallicity is a parameter which cannot be constrained independently, but can be determined from spectroscopic analysis. We have also compared our $[\mathrm{Fe} / \mathrm{H}]$ determination with spectroscopically determined metallicity estimates from Terrien et al. (2015; see Figs. 10 and 11). Terrien et al. (2015) used both $J$, $H$, and $K_{\mathrm{s}}$ band calibration given by Mann et al. (2013b) and the combinations of EW that effectively trace stellar metallicity from the $H$-band spectra given by Newton et al. (2014). Terrien et al. (2015) estimated the metallicities of the M dwarfs using the EW of the $\mathrm{NaI}$ feature at $2.2 \mu \mathrm{m}$ in the $K$ band of Infrared Telescope Facility (IRTF) spectra as used by Newton et al. (2014). We find an average deviation of 0.2 to $0.4 \mathrm{dex}$ in $[\mathrm{Fe} / \mathrm{H}]$ from Newton et al. (2014) and Terrien et al. (2015). A possible explanation for this deviation could be differences in the determination of $T_{\text {eff }}$. The $[\mathrm{Fe} / \mathrm{H}]$ and $T_{\text {eff }}$ are dependent to the point where there is normally a degeneracy of models based on this interdependence. The different parameter combinations of $T_{\text {eff }}$ and $\log g$ can produce the same $[\mathrm{Fe} / \mathrm{H}]$ at low resolution. These deviations could also be due to the fact that the BTSettl model provides a better description of the $\mathrm{M}$ dwarf atmospheres and therefore more accurate metallicities can be derived than when using other methods, which is also pointed out by Lindgren \& Heiter (2017).

The recent improvement in the BT-Settl model atmosphere could have implications beyond those noted in this study. The description of various physical process at these low temperatures is well explained by BT-Settl models. These models now provide a better fit to the high-resolution spectroscopic observations of $\mathrm{M}$ dwarfs and help in accurately determining their atmospheric parameters. To address our offset in metallicity using different sets of model atmospheres, we also made a comparison study with the MARCS model. This comparison suggests that the BT-Settl models describe cool atmospheres more accurately than the MARCS model. We plan to use our method along 

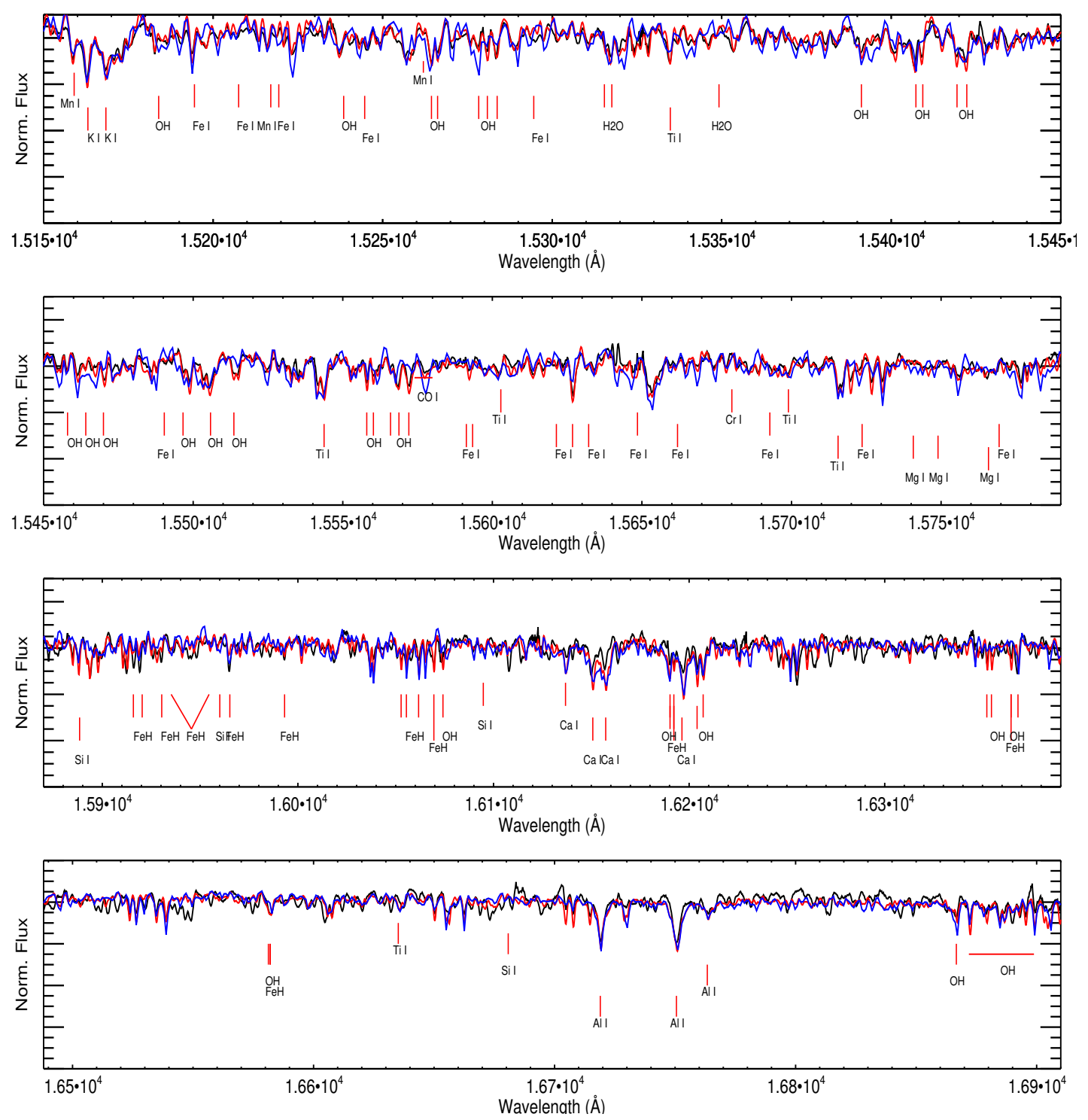

Fig. 9. APOGEE spectra of 2M08501918+1056436 (black) of spectral type M5.0 is compared with the best-fit BT-Settl (red) and MARCS model (blue). The best fit value for $T_{\text {eff }}, \log g$, and $[\mathrm{Fe} / \mathrm{H}]$ is $3100 / 5.5 /-0.0$.

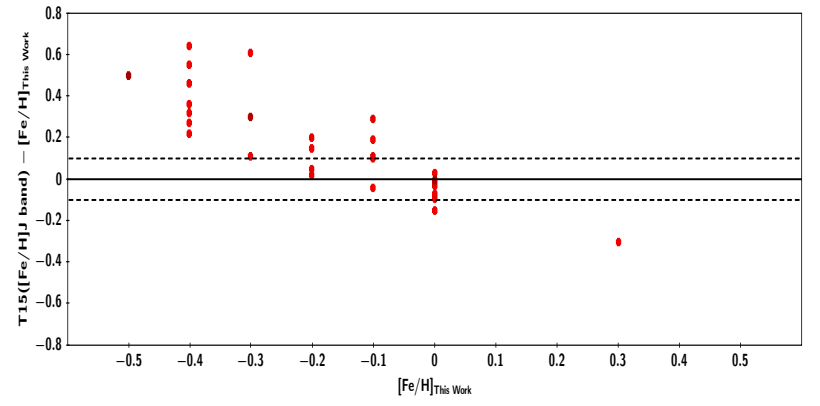

Fig. 10. Difference between the $[\mathrm{Fe} / \mathrm{H}]$ calibrations from Terrien et al. (2015, T15), estimated for the $\mathrm{M}$ dwarfs from $H$-band relationships given by Newton et al. (2014) calibrations and $[\mathrm{Fe} / \mathrm{H}]$ from this work. On the horizontal axis we show the $[\mathrm{Fe} / \mathrm{H}]$ that we infer from our best fit BT-Settl model used in this work. The black full line represents the origin and the dashed black lines represent the error from the grid size of 0.1 dex.

with the grid of these new BT-Settl models to estimate the stellar parameter of $\mathbf{M}$ dwarfs both in optical and in NIR spectra and photometry simultaneously to minimize the differences. The improvements in BT-Settl (Allard et al. 2013), achieved with the revision of solar abundances by Asplund et al. (2009) and Caffau et al. (2011), and by including updated atomic and molecular line opacities that dominate both in the optical and NIR range $M$ dwarfs, greatly help to reproduce the extensive and complex molecular absorption band systems present in $\mathrm{M}$ dwarf atmospheres.

As compared to other models, the line strength and shape of various atomic and molecular features is very well reproduced by the BT-Settl models but there is still need for improvement in the regions where the fit is not good. This could be due to the lack of various opacity line lists in the model; in particular the $\mathrm{FeH}$ line list is missing in the $H$ bandpass. Currently, the ExoMol group is developing an accurate and complete line list for $\mathrm{TiO}$ which is the next step to including them in the BT-Settl model before computing detailed model atmosphere grids and interior and evolution models at finer steps in the atmospheric parameters. The three-dimensional radiative hydrodynamics simulations and radiative transfer will help in understanding the effects of temperature inhomogeneities in the atmosphere which begin to have greater impact on the spectrum formation. 

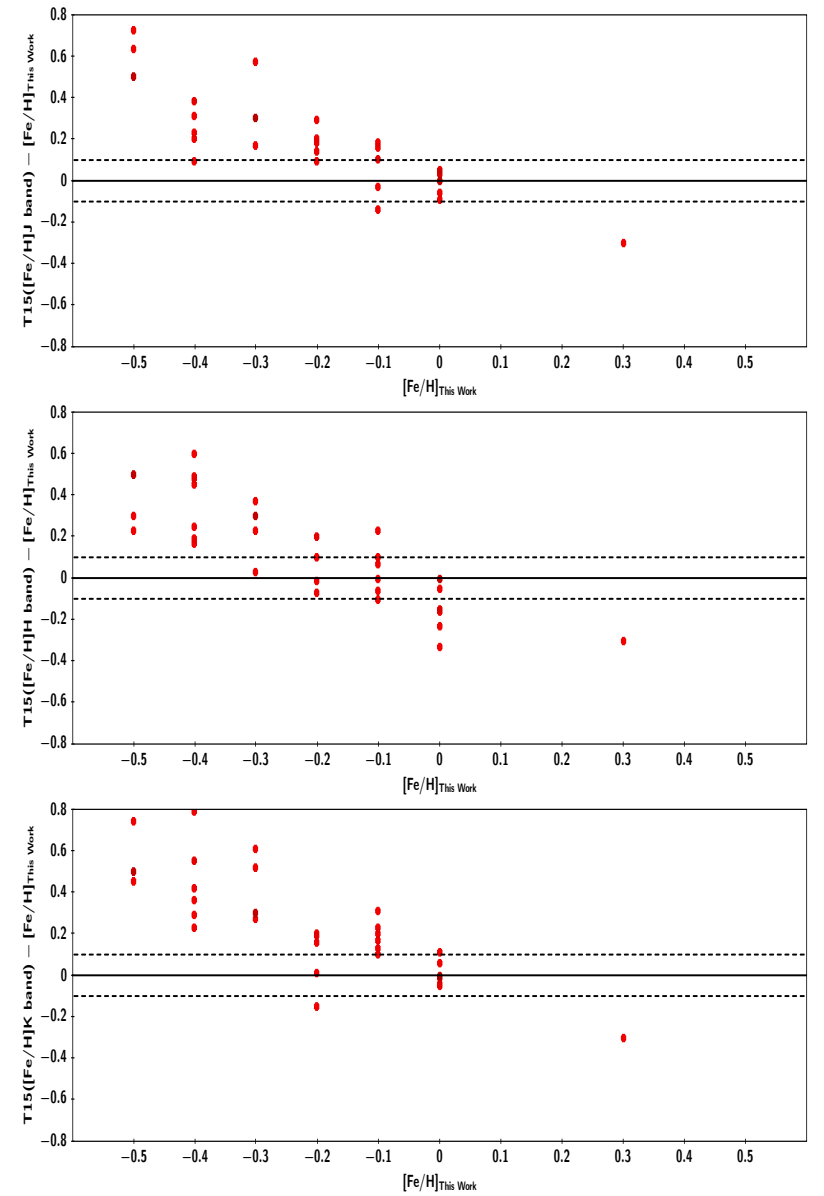

Fig. 11. Difference between the $[\mathrm{Fe} / \mathrm{H}]$ calibrations from Terrien et al. (2015, T15), estimated for the M dwarfs from Mann et al. (2013b) $J$ (top), $H$ (middle), and $K_{\mathrm{s}}$ (bottom) calibrations and $[\mathrm{Fe} / \mathrm{H}]$ from this work. On the horizontal axis we show the $[\mathrm{Fe} / \mathrm{H}]$ that we infer from our best fit BT-Settl model used in this work. The black full line represents the origin and the dashed black lines represent the error from the grid size of 0.1 dex.

Acknowledgements. The research leading to these results has received funding from the French "Programme National de Physique Stellaire" and the Programme National de Planétologie of CNRS (INSU). The computations were performed at the Pôle Scientifique de Modélisation Numérique (PSMN) at the École Normale Supérieure (ENS) in Lyon, and at the Gesellschaft für Wissenschaftliche Datenverarbeitung Göttingen in collaboration with the Institut für Astrophysik Göttingen. D.H. is supported by Sonderforschungsbereich SFB 881 "The Milky Way System" (subproject A4) of the German Research Foundation (DFG). G. D. C. Teixeira acknowledges support from the fellowship PD/BD/113478/2015 funded by FCT (Portugal) and POPH/FSE (EC). This work was supported in part by Fundação para a Ciência e a Tecnologia (FCT) through national funds (UID/FIS/04434/2013) and by FEDER through COMPETE2020 (POCI-01-0145-FEDER-007672). We also want to thank the anonymous referee for useful comments, which helped improve the paper. Funding for the Sloan Digital Sky Survey IV has been provided by the Alfred P. Sloan Foundation, the US Department of Energy Office of Science, and the Participating Institutions. SDSS-IV acknowledges support and resources from the Center for High-Performance Computing at the University of Utah. The SDSS web site is www.sdss.org. SDSS-IV is managed by the Astrophysical Research Consortium for the Participating Institutions of the SDSS Collaboration including the Brazilian Participation Group, the Carnegie Institution for Science, Carnegie Mellon University, the Chilean Participation Group, the French Participation Group, Harvard-Smithsonian Center for Astrophysics, Instituto de Astrofisica de Canarias, The Johns Hopkins University, Kavli Institute for the Physics and Mathematics of the Universe (IPMU)/University of Tokyo, Lawrence Berkeley National Laboratory, Leibniz Institut für Astrophysik Potsdam (AIP), Max-Planck-Institut für Astronomie (MPIA Heidelberg), MaxPlanck-Institut für Astrophysik (MPA Garching), Max-Planck-Institut fürExtraterrestrische Physik (MPE), National Astronomical Observatory of China,
New Mexico State University, New York University, University of Notre Dame, Observatório Nacional/MCTI, The Ohio State University, Pennsylvania State University, Shanghai Astronomical Observatory, United Kingdom Participation Group, Universidad Nacional Autónoma de México, University of Arizona, University of Colorado Boulder, University of Oxford, University of Portsmouth, University of Utah, University of Virginia, University of Washington, University of Wisconsin, Vanderbilt University, and Yale University.

\section{References}

Adibekyan, V. Z., Figueira, P., Santos, N. C., et al. 2013, A\&A, 554, A44 Alam, S., Albareti, F. D., Allende Prieto, C., et al. 2015, ApJS, 219, 12

Allard, F. 1990, Ph.D. Thesis, Ruprecht Karls Univ. Heidelberg

Allard, F., \& Hauschildt, P. H. 1995, ApJ, 445, 433

Allard, F., Hauschildt, P. H., Alexander, D. R., \& Starrfield, S. 1997, ARA\&A, 35,137

Allard, F., Alexander, D. R., \& Hauschildt, P. H. 1998, in Cool Stars, Stellar Systems, and the Sun, eds. R. A. Donahue \& J. A. Bookbinder, ASP Conf. Ser., 154, 63

Allard, F., Hauschildt, P. H., Alexander, D. R., Tamanai, A., \& Schweitzer, A. 2001, ApJ, 556, 357

Allard, F., Homeier, D., \& Freytag, B. 2012, Roy. Soc. London Philos. Trans. Ser. A, 370, 2765

Allard, F., Homeier, D., Freytag, B., et al. 2013, Mem. Soc. Astron. It. Suppl., 24, 128

Anglada-Escudé, G., Amado, P. J., Barnes, J., et al. 2016, Nature, 536, 437

Asplund, M., Grevesse, N., Sauval, A. J., \& Scott, P. 2009, ARA\&A, 47, 481

Baraffe, I., Homeier, D., Allard, F., \& Chabrier, G. 2015, A\&A, 577, A42

Bayo, A., Rodrigo, C., Barrado, D., \& Allard, F. 2014, Mem. Soc. Astron. It., 85,773

Bayo, A., Barrado, D., Allard, F., et al. 2017, MNRAS, 465, 760

Bean, J. L., Benedict, G. F., \& Endl, M. 2006a, ApJ, 653, L65

Bean, J. L., Sneden, C., Hauschildt, P. H., Johns-Krull, C. M., \& Benedict, G. F. 2006b, ApJ, 652, 1604

Bessell, M. S. 1991, AJ, 101, 662

Bochanski, J. J., Hawley, S. L., Covey, K. R., et al. 2010, AJ, 139, 2679

Bonfils, X., Forveille, T., Delfosse, X., et al. 2005, A\&A, 443, L15

Bonfils, X., Gillon, M., Udry, S., et al. 2012, A\&A, 546, A27

Boyajian, T. S., von Braun, K., van Belle, G., et al. 2012, ApJ, 757, 112

Brott, I., \& Hauschildt, P. H. 2005, in The Three-Dimensional Universe with Gaia, eds. C. Turon, K. S. O'Flaherty, \& M. A. C. Perryman, ESA SP, 576, 565

Burgasser, A. J., \& Kirkpatrick, J. D. 2006, ApJ, 645, 1485

Caffau, E., Ludwig, H.-G., Steffen, M., Freytag, B., \& Bonifacio, P. 2011, Sol. Phys., 268, 255

Casagrande, L., Flynn, C., \& Bessell, M. 2008, MNRAS, 389, 585

Chabrier, G., Baraffe, I., Allard, F., \& Hauschildt, P. 2000, ApJ, 542, 464

Cool, A. M., Piotto, G., \& King, I. R. 1996, ApJ, 468, 655

Deshpande, R., Blake, C. H., Bender, C. F., et al. 2013, AJ, 146, 156

Edvardsson, B., Andersen, J., Gustafsson, B., et al. 1993, A\&A, 275, 101

Fuhrmann, K. 1998, A\&A, 338, 161

García Pérez, A. E., Allende Prieto, C., Holtzman, J. A., et al. 2016, AJ, 151, 144

Gillon, M., Triaud, A. H. M. J., Demory, B.-O., et al. 2017, Nature, 542, 456

Gizis, J. E. 1997, AJ, 113, 806

Gould, A., Bahcall, J. N., \& Flynn, C. 1996, ApJ, 465, 759

Gratton, R. G., Carretta, E., \& Castelli, F. 1996, A\&A, 314, 191

Green, P. J., \& Margon, B. 1994, ApJ, 423, 723

Gustafsson, B., Edvardsson, B., Eriksson, K., et al. 2008, A\&A, 486, 951

Hauschildt, P. H., Baron, E., \& Allard, F. 1997, ApJ, 483, 390

Helling, C., Ackerman, A., Allard, F., et al. 2008, MNRAS, 391, 1854

Henry, T. J. 1998, in Brown Dwarfs and Extrasolar Planets, eds. R. Rebolo,

E. L. Martin, \& M. R. Zapatero Osorio, ASP Conf. Ser., 134, 28

Johnson, J. A., \& Apps, K. 2009, ApJ, 699, 933

Leggett, S. K., Allard, F., Berriman, G., Dahn, C. C., \& Hauschildt, P. H. 1996, ApJS, 104, 117

Leggett, S. K., Allard, F., \& Hauschildt, P. H. 1998, ApJ, 509, 836

Leggett, S. K., Allard, F., Dahn, C., et al. 2000, ApJ, 535, 965

Leggett, S. K., Allard, F., Geballe, T. R., Hauschildt, P. H., \& Schweitzer, A. 2001, ApJ, 548, 908

Lindgren, S., \& Heiter, U. 2017, A\&A, 504, A97

Majewski, S. R., Schiavon, R. P., Frinchaboy, P. M., et al. 2017, AJ, 154, 94

Mann, A. W., Brewer, J. M., Gaidos, E., Lépine, S., \& Hilton, E. J. 2013a, Astron.

Nachr., 334, 18

Mann, A. W., Gaidos, E., \& Ansdell, M. 2013b, ApJ, 779, 188

Mann, A. W., Deacon, N. R., Gaidos, E., et al. 2014, AJ, 147, 160 
Mann, A. W., Feiden, G. A., Gaidos, E., Boyajian, T., \& von Braun, K. 2015, ApJ, 804, 64

Mera, D., Chabrier, G., \& Baraffe, I. 1996, ApJ, 459, L87

Mészáros, S., Allende Prieto, C., Edvardsson, B., et al. 2012, AJ, 144, 120

Newton, E. R., Charbonneau, D., Irwin, J., et al. 2014, AJ, 147, 20

Newton, E. R., Charbonneau, D., Irwin, J., \& Mann, A. W. 2015, ApJ, 800, 85

Nidever, D. L., Holtzman, J. A., Allende Prieto, C., et al. 2015, AJ, 150, 173

Passegger, V. M., Wende-von Berg, S., \& Reiners, A. 2016, A\&A, 587, A19

Rajpurohit, A. S., Reylé, C., Schultheis, M., et al. 2012, A\&A, 545, A85

Rajpurohit, A. S., Reylé, C., Allard, F., et al. 2013, A\&A, 556, A15

Rajpurohit, A. S., Reylé, C., Allard, F., et al. 2014, A\&A, 564, A90

Rajpurohit, A. S., Reylé, C., Allard, F., et al. 2016, A\&A, 596, A33

Reiners, A. 2005, Astron. Nachr., 326, 930

Reiners, A., Mrotzek, N., Lemke, U., Hinrichs, J., \& Reinsch, K. 2016, A\&A, 587, A65

Renzini, A., Bragaglia, A., Ferraro, F. R., et al. 1996, ApJ, 465, L23

Rojas-Ayala, B., Covey, K. R., Muirhead, P. S., \& Lloyd, J. P. 2010, ApJ, 720, L113

Rojas-Ayala, B., Covey, K. R., Muirhead, P. S., \& Lloyd, J. P. 2012, ApJ, 748, 93
Ruiz, J. 1997, Earth Moon and Planets, 77, 99

Schlaufman, K. C., \& Laughlin, G. 2010, A\&A, 519, A105

Schmidt, S. J., Wagoner, E. L., Johnson, J. A., et al. 2016, MNRAS, 460, 2611

Ségransan, D., Kervella, P., Forveille, T., \& Queloz, D. 2003, A\&A, 397, L5

Souto, D., Cunha, K., García-Hernández, D. A., et al. 2017, ApJ, 835, 239

Terrien, R. C., Mahadevan, S., Bender, C. F., et al. 2012, ApJ, 747, L38

Terrien, R. C., Mahadevan, S., Deshpande, R., \& Bender, C. F. 2015, ApJS, 220, 16

Tsuji, T., Ohnaka, K., \& Aoki, W. 1996a, A\&A, 305, L1

Tsuji, T., Ohnaka, K., Aoki, W., \& Nakajima, T. 1996b, A\&A, 308, L29

Valenti, J. A., Piskunov, N., \& Johns-Krull, C. M. 1998, ApJ, 498, 851

Veeder, G. J. 1974, AJ, 79, 1056

Wilson, J. C., Hearty, F., Skrutskie, M. F., et al. 2010, in Ground-based and Airborne Instrumentation for Astronomy III, Proc. SPIE, 7735, $77351 \mathrm{C}$

Wilson, J. C., Hearty, F., Skrutskie, M. F., et al. 2012, in Ground-based and Airborne Instrumentation for Astronomy IV, Proc. SPIE, 8446, 84460

Wing, R. F., \& Rinsland, C. P. 1979, AJ, 84, 1235

Woolf, V. M., \& Wallerstein, G. 2006, PASP, 118, 218

Woolf, V. M., Lépine, S., \& Wallerstein, G. 2009, PASP, 121, 117 